\title{
Application of Virtual Reality Technology in Training of Cardiopulmonary Resuscitation Skills for Nursing Student
}

\author{
Xiuxian Mo \\ (Guangxi University of Science and Technology, Liuzhou, Guangxi, 545006)
}

\begin{abstract}
To study the application value of virtual reality technology in the training of cardiopulmonary resuscitation skills for nursing students, and to provide a more efficient way to optimize the training of nursing students' skills. Method: The nursing students in two classes of school nursing major were selected as the research object. The control group adopted the scenario simulation method for training CPR skills. The experimental group adopted virtual reality technology for training. The total scores of CPR skills and the scores of key items at different time points were compared. Result: The full scores of CPR skills and the scores of key items in the experimental group were significantly higher than those in the control group at the completion of the training, one month after the training and six months after the training $(\mathrm{P}<0$. 05). Conclusion: Virtual reality technology contributed a more significant effect on CPR skills training, and the skill level was maintained for a longer time.
\end{abstract}

Keywords : virtual reality technology; school nursing students; cardiopulmonary resuscitation; skills training; application effect

DOI : 10.36012 / fhe. v2i2. 2768

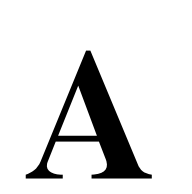

$\mathrm{s}$ a commonly used skill in medical treatment, cardiopulmonary resuscitation is also a kind of basic first aid ability that medical staffs need to master ${ }^{[1]}$. After skills training, the skill level of medical staff will gradually decline over time, which is closely related to the actual operation of cardiopulmonary resuscitation in the study and work of medical staff. For nursing students at school, receiving CPR skills training has a significant effect on their skill level and its maintenance ${ }^{[2]}$. As a relatively new type of computer technology, the specific equipment used in virtual reality technology can enable users to experience the virtual world. The user's virtual experience is verisimilar under the influence of sense organs ${ }^{[3]}$. The application of virtual reality technology in the teaching procedure of medical education can improve students' skills training and have a positive effect on training effectiveness and skills maintenance. The biggest advantage of virtual reality technology is the sense of realism and imagination. With the help of visual, auditory, and other senses, as well as supporting equipment, students can "really" experience the scene of patient rescue and acquire corresponding knowledge and skills during the rescue process. Under the effect of virtual reality technology, students will form a reasonable and rational cognition of CPR skills. They will understand, master and remember CPR skills in depth, which will extend the period of CPR training.

From May 2018 to November 2018, the nurs- 
ing students in two classes of school nursing major were selected as the research object. After random grouping, scenario simulation method and virtual reality technology were used in CPR skills training. The total scores of CPR skills and the scores of key items were compared between both groups at the completion of the training, one month after the training and six months.

after the training. The application value of virtual reality technology in the training of cardiopulmonary resuscitation skills for nursing students was clarified to provide a more efficient way for the optimization of skills training of nursing students. The report is as follows:

\section{Data and Methods}

\section{1 General data}

From May 2018 to November 2018, the nursing students in two classes of school nursing major were selected as the research object. The number of people in each class was 30, the experimental and control groups were randomly set. Among the 30 nursing students in the experimental group, 10 were male nursing students, and 20 were female nursing students, the average age was $18.41 \pm 0$. 71 , and the average credit score was $82.34 \pm 3$. 64. Among the 30 nursing students in the control group, 9 were male nursing students, and 21 were female nursing students, the average age was 18 . $52 \pm 0.67$, and the average credit score was 82.21 \pm 3. 59. There was no significant difference in age, gender, or average credit score between both groups, which were comparable $(\mathrm{P}>0.05)$.

The admission criteria for nursing students were as follows: (1) Informed and agreed to this research experiment; (2) Did not receive CPR skills training before entering the group. Nursing students who were unable to complete CPR skills due to physical reasons, nursing students who took leave during the study period or failed to complete follow - up observation were excluded.

\section{2 Methods}

In CPR skills training, the "2015 version of CPR theory and single - person operation skills" was used. The two groups received the same theoretical lecture for half an hour, after which they would be trained separately for 4 hours.

The control group adopted the scenario simulation method for training CPR skills. The teacher explained and demonstrated CPR operation; the students carried out simulation exercises on the model to simulate the situation of out - of - hospital cardiac arrest and performed CPR following standard operation requirements. The teacher observed and provided guidance during practice and pointed out and corrected errors in time.

The experimental group adopted virtual reality technology for training. The teacher explained and demonstrated the CPR operation. After that, students practiced CPR on the model with virtual reality glasses, which were installed with CPR training software in advance, so students could $\mathrm{di}^{-}$ rectly experience the situation of out - of - hospital cardiac arrest and performed CPR accordingly. In virtual reality mode, the first - aid process included 27 theoretical questions and five cyclic operations. When the out - of - hospital emergency scene developed to a certain degree, relevant theoretical questions would appear, and only when students answered the questions correctly would the scene continue to proceed, the ambulance would arrive at the scene when the first aid was completed. Teachers also observed and guided the whole process.

\section{3 observation target}

As examiners, two nurses would evaluate the total score of CPR skills and the score of key items respectively at the completion of the training, one month after the training and six months after the training.

\section{4 statistical treatments}

For the measurement and statistical data in this study, SPSS19. 0 statistical software was used for calculation and processing. Enumeration data was tested and verified by $\left(x^{2}\right)$, among which the 
measurement data was expressed by $(\bar{x} \pm s)$ and verified by $(\mathrm{t}) . \mathrm{P}<0.05$ meant the difference was statistically significant.

\section{Result}

\section{1 Comparison of total scores of CPR skills between two} groups at different time points after training

As shown in Table 1 , the total scores of CPR skills in the experimental group were significantly higher than those of the control group at the completion of the training, one month after the training and six months after the training $(\mathrm{P}<0.05)$.

Table 1 Comparison of total scores of CPR skills between two groups at different time points after training $(\bar{x} \pm s$, score $)$

\begin{tabular}{c|c|c|c|c}
\hline Group & $\begin{array}{c}\text { Case } \\
\text { number }\end{array}$ & $\begin{array}{c}\text { at the completion } \\
\text { of the training }\end{array}$ & $\begin{array}{c}\text { One month after } \\
\text { the training }\end{array}$ & $\begin{array}{c}\text { Six months after } \\
\text { the training }\end{array}$ \\
\hline $\begin{array}{c}\text { experimental } \\
\text { group }\end{array}$ & 30 & $95.43 \pm 4.30$ & $92.34 \pm 7.76$ & $88.10 \pm 10.34$ \\
\hline $\begin{array}{c}\text { control group } \\
\mathrm{t}\end{array}$ & 30 & $83.89 \pm 6.41$ & $82.80 \pm 7.64$ & $80.34 \pm 9.25$ \\
\hline $\mathrm{P}$ & & 14.221 & 12.954 & 12.014 \\
\hline
\end{tabular}

\section{2 Comparison of the scores of key items between} two groups at different time points after training

As shown in Table 2 , the scores of key items in the experimental group were significantly higher than those of the control group at the completion of the training, one month after the training and six months after the training $(\mathrm{P}<0.05)$.

Table 2 Comparison of the scores of key items between two groups at different time points after training $(\bar{x} \pm s$, score $)$

\begin{tabular}{c|c|c|c|c}
\hline Group & $\begin{array}{c}\text { Case } \\
\text { number }\end{array}$ & $\begin{array}{c}\text { at the completion } \\
\text { of the training }\end{array}$ & $\begin{array}{c}\text { One month after } \\
\text { the training }\end{array}$ & $\begin{array}{c}\text { Six months after } \\
\text { the training }\end{array}$ \\
\hline $\begin{array}{c}\text { experimental } \\
\text { group }\end{array}$ & 30 & $19.54 \pm 0.71$ & $18.86 \pm 1.54$ & $18.29 \pm 2.18$ \\
\hline control group & 30 & $16.12 \pm 2.42$ & $15.97 \pm 3.09$ & $15.62 \pm 3.40$ \\
\hline $\mathrm{t}$ & & 12.984 & 12.246 & 10.954 \\
\hline $\mathrm{P}$ & & 0.024 & 0.026 & 0.029 \\
\hline
\end{tabular}

\section{Discussions}

Cardiopulmonary resuscitation is a commonly used first - aid method. Strengthening the cardiopulmonary resuscitation skills of nursing students in school has positive significance for improving teaching effectiveness and students' professional skills. The results of this study shows: the total scores of CPR skills in the experimental group were significantly higher than those of the control group at the completion of the training, one month after the training and six months after the training $(\mathrm{P}<0.05)$; the scores of key items in the experimental group were significantly higher than those of the control group at the completion of the training, one month after the training and six months after the training $(\mathrm{P}<0.05)$. The application of virtual reality technology in CPR skills training can significantly improve students' skill levels. Highquality cardiopulmonary resuscitation needs to be ensured in terms of press rate and depth. The thoracic cage needs to be fully rebound after pressing, which requires that interruption and hyperventilation be avoided as far as possible during a press ${ }^{[4]}$. The implementation of high - quality cardiopulmonary resuscitation is an important condition for improving the success rate in the therapy of sudden cardiac arrest, which requires that medical staff maintain a high - level cardiopulmonary resuscitation skill ${ }^{[5]}$. In this study, the fact that nursing students who received CPR skills training with virtual reality technology had higher total scores and scores of key items proves that the application of virtual reality technology in CPR skills training can not only ensure that students master related skills but also help maintain their skills. The application of virtual reality technology in CPR skills training helps significantly improve students 'attention and concentration, increases students' interest in skills training, and guarantees the effect of skills training.

As emerging computer technology, virtual reality technology can effectively attract students into skill training and satisfy their curiosity and feeling of freshness ${ }^{[6]}$. At the same time, virtual reality technology can provide students with very realistic and vivid scenarios to help them better perform in CPR training, maintain a high-level concentration, get a deeper impression of skills training, and maintain a high - level CPR skill for a 
longer period of time. As time goes by, students will gradually forget what they used to learn, which is undeniable. Psychology experts point out that forgetting in the learning process is fast initially but slow afterward ${ }^{[7-8]}$. The same is true for skills training. If regular intensive training is not performed after initial training, the nursing skills of students will gradually decline. Compared with conventional situational teaching, virtual reality technology provides students with real ambulance scenes, which help students almost wholly, enter the natural ambulance environment. From the perspective of situational cognitive theory, some researchers believe that skill training needs to be conducted in a scene similar to the actual one and aims to solve real problems. This can not only ensure the improvement and maintenance of students' skill levels but also truly cultivate talents needed by society ${ }^{[9-10]}$. Sudden cardiac arrest is an emergency in clinical treatment, so it is impossible to carry out teaching activities in the rescue process. Therefore, traditional teaching often adopts scenario simulation and models to guide students to carry out skill training. Still, the training effect is limited as the authenticity of the scene is relatively limited. In summary, in the training of cardiopulmonary resuscitation skills, the reasonable use of virtual reality technology can obtain more significant training effects. The full score of skills and scores of key items are significantly improved, the skill level is maintained for a long time, and the time for skill retraining is extended, which is conducive to the improvement and optimization of medical education methods.

\section{References}

[1] Wang Siyao, Zhang Huan, Peng Rui, etc. Application of Virtual Reality Technology in Training of Cardiopulmonary Resuscitation Skills for Medical Students [J]. Chinese Journal of Modern Nursing, 2018, 24 (36) : $4455-4458$.
[2] Huang Feng, Wang Wei, Luo Zuchun. Application Research of Virtual Simulation Teaching Platform in Practical Teaching of Cardiopulmonary Resuscitation Technology [J]. Journal of Snake, 2019, 10 (2): 288 -289 .

[3] Yang wen, Shu Xiaogang, Yang Guangyao, etc. The Application of Flipped Classroom Teaching Method Based on WeChat Platform in Clinical Skill Teaching [J]. Chinese Journal of Medical Education, 2019, 39 (4) $: 280$.

[4] Cao Yimei, Wang Jing, Qin Jian, etc. The Application of CBL Combined with Physiologically Driven High Simulated Simulator in the Training of Resident CPR Non-technical Ability [J]. China Journal of Emergency Resuscitation and Disaster Medicine, 2018 (12) : $1238-1239$.

[5] Yin Jun. Application of Modern Information Technology in Cardiopulmonary Resuscitation Teaching $[\mathrm{J}]$. Modern vocational education,2019,7(9):148-149.

[6] Shi min, Lin Yuhua, Li Jinjun, etc. Application of Virtual Reality Technology in Practical Teaching of Dental Practice [J]. Chinese Journal of Dental Materials and Devices,2019,10(2):53-56.

[7] Lam J F, Gosselin L, Rushton P W. The Use of Virtual Technology as an Intervention for Wheelchair Skills Training: A Systematic Review[J]. Archives of Physical Medicine and Rehabilitation, 2018:S000399931830159X.

[8] Pettersen T R, Jan Martensson, Asa Axelsson, et al. European cardiovascular nurses' and allied professionals' knowledge and practical skills regarding cardiopulmonary resuscitation[J]. European Journal of Cardiovascular Nursing Journal of the Working Group on Cardiovascular Nursing of the European Society of Cardiology, 2018, 17(4):1474515117745298.

[9] Ma gorzata migrodzka. Development of Virtual Reality Technology in the Aspect of Educational Applications [J]. Nephron Clinical Practice, 2017, 26 (4): 117 -133 .

[10] Spangenberg T, Schewel J, Dreher A, et al. Health - related quality of life after extracorporeal cardiopulmonary resuscitation in refractory cardiac arrest $[\mathrm{J}]$. Resutation, 2018, 127:73-78. 